\title{
Learning to play $K$-armed bandit problems
}

\author{
Francis Maes ${ }^{1}$, Louis Wehenkel ${ }^{1}$ and Damien Ernst ${ }^{1}$ \\ ${ }^{1}$ University of Liège \\ Dept. of Electrical Engineering and Computer Science \\ Institut Montefiore, B28, B-4000, Liège - Belgium \\ francis.maes@ulg.ac.be,L.Wehenkel@ulg.ac.be,dernst@ulg.ac.be
}

Keywords: Multi-armed bandit problems, reinforcement learning, exploration-exploitation dilemma

\begin{abstract}
We propose a learning approach to pre-compute K-armed bandit playing policies by exploiting prior information describing the class of problems targeted by the player. Our algorithm first samples a set of K-armed bandit problems from the given prior, and then chooses in a space of candidate policies one that gives the best average performances over these problems. The candidate policies use an index for ranking the arms and pick at each play the arm with the highest index; the index for each arm is computed in the form of a linear combination of features describing the history of plays (e.g., number of draws, average reward, variance of rewards and higher order moments), and an estimation of distribution algorithm is used to determine its optimal parameters in the form of feature weights. We carry out simulations in the case where the prior assumes a fixed number of Bernoulli arms, a fixed horizon, and uniformly distributed parameters of the Bernoulli arms. These simulations show that learned strategies perform very well with respect to several other strategies previously proposed in the literature (UCB1, UCB2, UCB-V, KL-UCB and $\varepsilon_{n}$-GREEDY); they also highlight the robustness of these strategies with respect to wrong prior information.
\end{abstract}

\section{INTRODUCTION}

The exploration versus exploitation dilemma arises in many fields such as finance, medicine, engineering as well as artificial intelligence. The finite horizon $K$-armed bandit problem formalizes this dilemma in the most simple form (Robbins, 1952): a gambler has $T$ coins, and at each step he may choose among one of $K$ slots (or arms) to allocate one of these coins, and then earns some money (his reward) depending on the response of the machine he selected. Rewards are sampled from an unknown probability distribution dependent on the selected arm but otherwise independent of previous plays. The goal of the gambler is simply to collect the largest cumulated reward once he has exhausted his coins (i.e. after $T$ plays). A rational player knowing the reward distributions of the $K$ arms would play at every stage an arm with maximal expected reward, so as to maximize his expected cumulative reward (irrespectively of the number $K$ of arms and the number of $T$ coins). When the reward distributions are unknown, it is however much less trivial to decide how to play optimally.

Most theoretical works that have studied the $K$ armed bandit problem have focused on the design of generic policies which are provably optimal in asymptotic conditions (large $T$ ), independently of the number of arms $K$, and assuming only some very unrestrictive regularity conditions on the reward distributions (e.g., bounded support). Among these, some work by computing at every play a quantity called "upper confidence index" for each arm that depends on the rewards collected so far by this arm, and by selecting for the next play (or round of plays) the arm with the highest index. Such index-based policies have been initially introduced by (Lai and Robbins, 1985) where the indexes were difficult to compute. More easy to compute indexes where proposed later on (Agrawal, 1995; Auer et al., 2002; Audibert et al., 2007).

These "index based" policies typically involve meta-parameters whose values impact their relative performances. Usually, when reporting simulation results, authors manually tuned these values on problems that share similarities with their test problems (e.g., the same type of distributions for generating the rewards) by running trial-and-error simulations (Auer et al., 2002; Audibert et al., 2008). In doing this, they actually exploited prior information on the $K$-armed bandit problems to select the meta-parameters.

Starting from this observation, we elaborated a systematic approach for learning in an automatic way 
good policies for playing $K$-armed bandit problems. This approach explicitly exploits the prior information on the target set of $K$-armed bandit problems; it first generates a sample of problems compliant with this prior information and then searches in a parametric set of candidate policies one that yields optimal average performances over this sample. This approach allows to automatically tune meta-parameters of existing upper confidence indexes. But, more importantly, it opens the door for searching within a much broader class of policies one that is optimal for a given set of problems compliant with the prior information. In particular, we show, in the case of Bernoulli arms, that when the number $K$ of arms and the playing horizon $T$ are fully specified a priori, this approach may be very productive. We also evaluate the robustness of the learned policies with respect to erroneous prior assumptions.

After recalling in the next section elements related to the K-armed bandit problem, we present in details our approach and algorithms in Section 3. In Section 4 , we report some convincing simulation results. Finally, we will conclude in Section 5 and present future research directions.

\section{THE K-ARMED BANDIT PROBLEM}

We denote by $i \in\{1,2, \ldots, K\}$ the $(K \geq 2)$ arms of the bandit problem, by $v_{i}$ the reward distribution of $\operatorname{arm} i$, and by $\mu_{i}$ its expected value; $b_{t}$ is the arm played at round $t$, and $r_{t} \sim v_{b_{t}}$ is the obtained reward. $H_{t}=\left[b_{1}, r_{1}, b_{2}, r_{2}, \ldots, b_{t}, r_{t}\right]$ is a vector that gathers the history over the first $t$ plays, and we denote by $\mathcal{H}$ the set of all possible histories of any length $t$.

A policy $\pi: \mathcal{H} \rightarrow\{1,2, \ldots, K\}$ is an algorithm that processes at play $t$ the vector $H_{t-1}$ to select the arm $b_{t} \in\{1,2, \ldots, K\}$ :

$$
b_{t}=\pi\left(H_{t-1}\right) .
$$

The regret of the policy $\pi$ after $T$ plays is defined by:

$$
R_{T}^{\pi}=T \mu^{*}-\sum_{t=1}^{T} r_{t}
$$

where $\mu^{*}=\max _{k} \mu_{k}$ refers to the expected reward of the optimal arm. The expected value of the regret represents the expected loss due to the fact that the policy does not always play the best machine. It can be written as:

$$
\mathrm{E}\left\{R_{T}^{\pi}\right\}=\sum_{k=1}^{K} \mathrm{E}\left\{T_{k}(T)\right\}\left(\mu^{*}-\mu_{k}\right),
$$

where $T_{k}(T)$ denotes the number of times the policy has drawn arm $k$ on the first $T$ rounds.

The $K$-armed bandit problem aims at finding a policy $\pi^{*}$ that for a given $K$ minimizes the expected regret (or, in other words, maximizes the expected reward), ideally for any $T$ and any $\left\{\mathrm{v}_{i}\right\}_{i=1}^{K}$.

\section{DESCRIPTION OF OUR APPROACH}

We now introduce our approach for learning $K$ armed bandit policies given a priori information on the set of target problems. We also describe the fully specified instance of this approach that is tested in Section 4 and compared against several other policies introduced previously in the literature, namely UCB1, UCB2 (Auer et al., 2002), UCB-V (Audibert et al., 2007), $\varepsilon_{n}$-GREEDY (Sutton and Barto, 1998) and variants of these policies.

\subsection{Generic description}

In order to identify good bandit policies, we exploit prior knowledge on the problem to be solved represented as a distribution $\mathcal{D}_{P}$ over bandit problems $P=\left(v_{1}, \ldots, v_{K}\right)$. From this distribution, we can sample as many bandit problems as desired. In order to learn a policy exploiting this knowledge, we propose to proceed as follows. First, we sample a set of training bandit problems $P^{(1)}, \ldots, P^{(N)}$ from $\mathcal{D}_{P}$. This set should be large enough to be representative of the target space of bandit problems. We then select a parametric family of candidate policies $\Pi_{\Theta} \subset$ $\{1,2, \ldots, K\}^{\mathcal{H}}$ whose members are policies $\pi_{\theta}$ that are fully defined given parameters $\theta \in \Theta$. We finally solve the following optimization problem:

$$
\theta^{*}=\underset{\theta \in \Theta}{\operatorname{argmin}} \Delta\left(\pi_{\theta}\right)
$$

where $\Delta(\pi) \in \mathbb{R}$ is a loss function that computes the empirical mean of expected regret of $\pi$ on the training set:

$$
\Delta(\pi)=\frac{1}{N} \sum_{i=1}^{N} \mathrm{E}\left\{R_{T}^{\pi}\left(P^{(i)}\right)\right\}
$$

where $T$ is the (a-priori given) time playing horizon.

Note that since $\mathrm{E}\left\{R_{T}^{\pi}\right\}$ relies on the expected values of $T_{k}(T)$, computing $\Delta(\pi)$ exactly is impossible in the general case. We therefore estimate the $E\left\{T_{k}(T)\right\}$ quantities by averaging their observed values over a large number of simulations, as described in Section 4. 


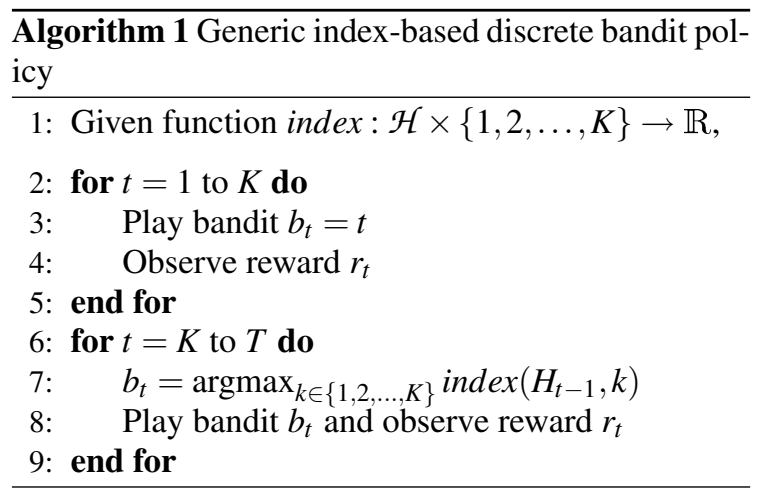

\subsection{Family of candidate policies considered}

As candidate policies we will consider policies which are fully defined by a scoring function index : $\mathcal{H} \times$ $\{1,2, \ldots, K\} \rightarrow \mathbb{R}$. These policies are sketched in Algorithm 1 and work as follows. During the first $K$ plays, they play sequentially the machines $1,2, \ldots, K$. In all subsequent plays, these policies compute for every machine $k$ the score index $\left(H_{t-1}, k\right) \in \mathbb{R}$ that depends on the arm $k$ and on the previous history $H_{t-1}$. At each step, the arm (or one of the arms) with the largest score is then selected.

Note that most well-known previously proposed bandit policies are particular cases of Algorithm 1. As an example, the policy UCB 1 (Auer et al., 2002) is an index-based bandit policy with:

$$
\operatorname{index}^{u c b 1}\left(H_{t}, k\right)=\bar{r}_{k}+\sqrt{\frac{2 \ln t}{T_{k}}},
$$

where $\bar{r}_{k}$ is the average reward obtained from machine $k$ and $T_{k}$ is the number of times machine $k$ has been played so far.

To define the parametric family of candidate policies $\Pi_{\Theta}$, we use index functions expressed as linear combinations of history features. These index functions rely on an history feature function $\phi: \mathcal{H} \times$ $\{1,2, \ldots, K\} \rightarrow \mathbb{R}^{d}$, that describes the history w.r.t. a given arm as a vector of scalar features. Each such scalar feature may describe any aspect of the history, including empirical reward moments, current time step, arm play counts or combinations of these variables.

Given the function $\phi(\cdot, \cdot)$, index functions are defined by

$$
\operatorname{index}_{\theta}\left(H_{t}, k\right)=\left\langle\theta, \phi\left(H_{t}, k\right)\right\rangle,
$$

where $\theta \in \mathbb{R}^{d}$ are parameters and $\langle\cdot, \cdot\rangle$ is the classical dot product operator.
The features computed by $\phi(\cdot, \cdot)$ should be relevant to the problem of assigning good indices to bandits. The set of features should not be too large to avoid optimization and learning difficulties related to the large number of parameters, but it should be large enough to provide the basis for a rich class of bandit policies. We propose one particular history feature function in Section 4 that can be used for any discrete bandit problem and that leads to successful policies.

The set of candidate policies $\Pi_{\Theta}$ is composed of all index-based policies obtained with index functions defined by Equation 7 given parameters $\theta \in \mathbb{R}^{d}$.

\subsection{Optimisation algorithm}

The optimization problem defined by Equation 4 is a hard optimization problem, with an objective function that has a complex relation with the parameters $\theta$. A slight change in the parameter vector $\theta$ may lead to significantly different bandit episodes and expected regret values. Local optimization approaches may thus not be appropriate here. Instead, we suggest the use of derivative-free global optimization algorithms.

In this work, we use a powerful, yet simple, class of global optimization algorithms known as Estimation of Distribution Algorithms (EDA) (Gonzalez et al., 2002). EDAs rely on a probabilistic model to describe promising regions of the search space and to sample good candidate solutions. This is performed by repeating iterations that first sample a population of $n_{p}$ candidates using the current probabilistic model and then fit a new probabilistic model given the $b<n_{p}$ best candidates.

Any kind of probabilistic model may be used inside an EDA. The simplest form of EDAs uses one marginal distribution per variable to optimize and is known as the univariate marginal distribution algorithm (Pelikan and Mühlenbein, 1998). We have adopted this approach by using one Gaussian distribution $\mathcal{N}\left(\mu_{p}, \sigma_{p}^{2}\right)$ for each parameter $\theta_{p}$. Although this approach is simple, it proved to be quite effective experimentally to solve Equation 4. The full details of our EDA-based policy learning procedure are given by Algorithm 2. The initial distributions are standard Gaussian distributions $\mathcal{N}(0,1)$. The policy that is returned corresponds to the $\theta$ parameters that led to the lowest observed value of $\Delta\left(\pi_{\theta}\right)$.

\section{NUMERICAL EXPERIMENTS}

The aim of this Section is to show that the expected regret can significantly be improved by ex- 


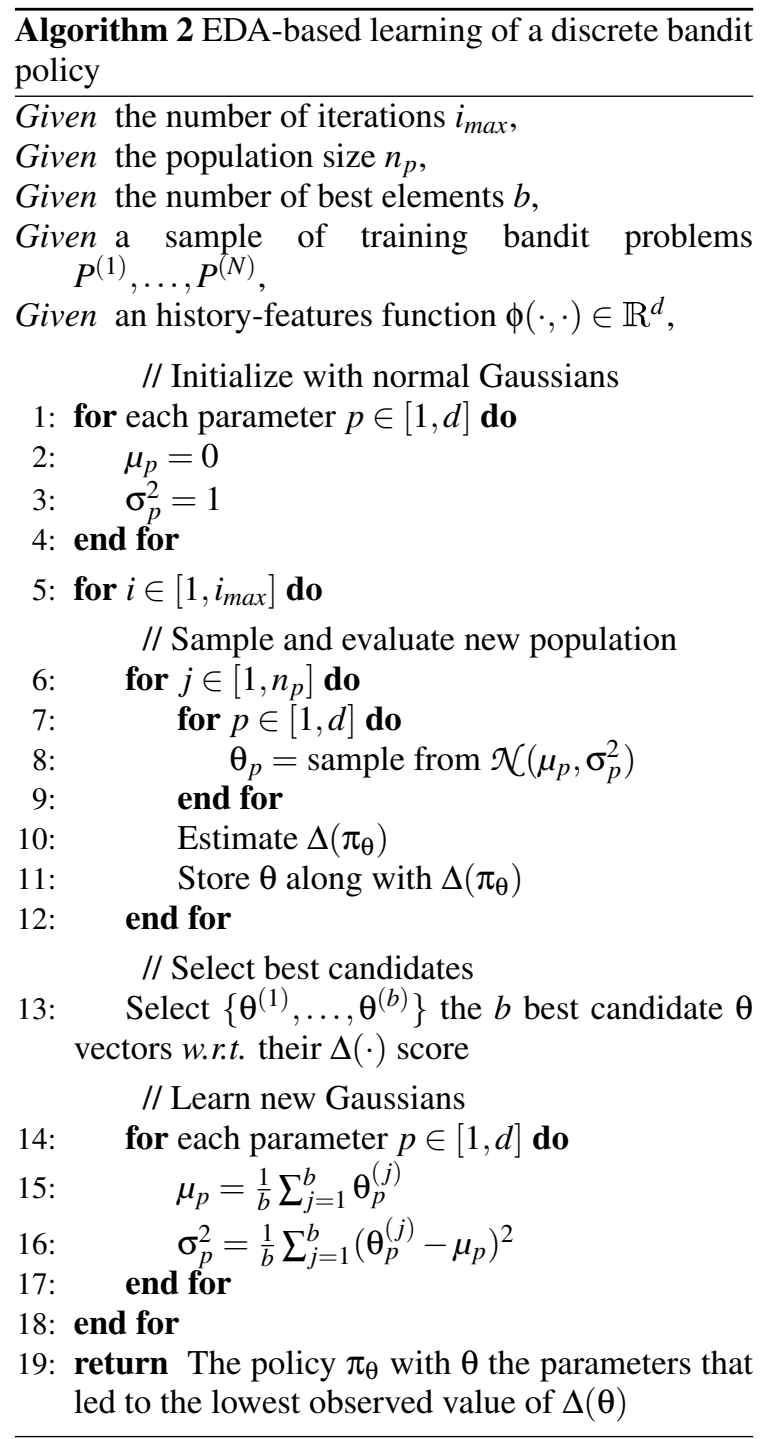

ploiting prior knowledge on bandit problems, and that learning general index-based policies with a large number of parameters outperforms careful tuning of all previously proposed simple bandit policies.

To illustrate our approach, we consider the scenario where the number of arms, the playing horizon and the kind of distributions $v_{i}$ are known a priori and where the parameters of these distributions is missing information. In particular, we focus on two-arms bandit problems with Bernoulli distributions whose expectations are uniformly drawn from $[0,1]$. Hence, in order to sample a bandit problem from $\mathcal{D}_{P}$, we draw the expectations $p_{1}$ and $p_{2}$ uniformly from $[0,1]$ and return the bandit problem defined by two-Bernoulli arms with $p_{1}$ and $p_{2}$.

Baselines. We consider the following baseline policies: the $\varepsilon_{n}$-GREEDY policy (Sutton and Barto,
1998) as described in (Auer et al., 2002), the policies introduced by (Auer et al., 2002): UCB 1, UCB 1BERNOULLI ${ }^{1}$, UCB1-NORMAL and UCB2, the policy KL-UCB introduced in (Garivier and Cappé, 2011) and the policy UCB-V proposed by (Audibert et al., 2007). Except $\varepsilon_{n}$-GREEDY, all these policies belong to the family of index-based policies discussed in the previous Section. UCB1-BERNOULLI and UCB 1-NORMAL are parameter-free policies designed for bandit problems with Bernoulli distributions and for problems with Normal distributions respectively. All the other policies have metaparameters that can be tuned to improve the quality of the policy. $\varepsilon_{n}$-GREEDY has two parameters $c>0$ and $0<d<1$, UCB2 has one parameter $0<\alpha<1$, KL-UCB has one parameter $c \geq 0$ and UCB-V has two parameters $\zeta>0$ and $c>0$. We refer the reader to (Auer et al., 2002; Audibert et al., 2007; Garivier and Cappé, 2011) for detailed explanations of these parameters. For the tunable version of UCB 1, we use the following formula:

$$
\operatorname{index}^{u c b 1(C)}\left(H_{t}, k\right)=\bar{r}_{k}+\sqrt{\frac{C \ln t}{T_{k}}}
$$

where $C>0$ is the meta-parameter to tradeoff between exploration and exploitation.

Tuning / training procedure. To make our comparison fair, we use the same tuning / training procedure for all the policies, which is Algorithm 2 with $i_{\text {max }}=100$ iterations, $n_{p}=\max (8 d, 40)$ candidate policies per iteration and $b=n_{p} / 4$ best elements, where $d$ is the number of parameters to optimize. Having a linear dependency between $n_{p}$ and $d$ is a classical choice when using EDAs (Rubenstein and Kroese, 2004). Note that, in most cases the optimization is solved in a few or a few tens iterations. Our simulations have shown that $i_{\max }=100$ is a careful choice for ensuring that the optimization has enough time to properly converge. For the baseline policies where some default values are advocated, we use these values as initial expectation of the EDA Gaussians. Otherwise, the initial Gaussians are centered on zero. Nothing is done to enforce the EDA to respect the constraints on the parameters (e.g., $c>0$ and $0<d<1$ for $\varepsilon_{n}$-GREEDY). In practice, the EDA automatically identifies interesting regions of the search space that respect these constraints.

History features function. We now detail the particular $\phi(\cdot, \cdot)$ function that we used in our exper-

\footnotetext{
${ }^{1}$ The original name of this policy is UCB-TUNED. Since this paper mostly deals with policies having parameters, we changed the name to UCB1-BERNOULLI to make clear that no parameter tuning has to be done with this policy.
} 
iments and that can be applied to any discrete bandit problem. To compute $\phi\left(H_{t}, k\right)$, we first compute the following four variables:

$$
v_{1}=\sqrt{\ln t} \quad v_{2}=\sqrt{\frac{1}{T_{k}}} \quad v_{3}=\bar{r}_{k} \quad v_{4}=\overline{\rho_{k}}
$$

i.e. the square root of the logarithm of the current time step, the inverse square root of the number of times arm $k$ has been played, the empirical mean and standard deviation of the rewards obtained so far by arm $k$.

Then, these variables are multiplied in different ways to produce features. The number of these combinations is controlled by a parameter $P$ whose default value is 1 . Given $P$, there is one feature $f_{i, j, k, l}$ per possible combinations of values of $i, j, k, l \in\{0, \ldots, P\}$, which is defined as follows:

$$
f_{i, j, k, l}=v_{1}^{i} v_{2}^{j} v_{3}^{k} v_{4}^{l}
$$

In the following, we denote POWER-1 (resp., POWER-2) the policy learned using function $\phi\left(H_{t}, k\right)$ with parameter $P=1$ (resp., $P=2$ ). The index function that underlies these policies can be written as following:

$$
\text { index }^{\text {power }}\left(H_{t}, k\right)=\sum_{i=0}^{P} \sum_{j=0}^{P} \sum_{k=0}^{P} \sum_{l=0}^{P} \theta_{i, j, k, l} v_{1}^{i} v_{2}^{j} v_{3}^{k} v_{4}^{l}
$$

where $\theta_{i, j, k, l}$ are the learned parameters. The POWER-1 policy has 16 such parameters and the POWER-2 has 81 parameters.

Training and testing sets. Since we are learning policies, care should be taken with generalization issues. As usual in supervised machine learning, we use a training set which is distinct from the testing set. The training set is composed of $N=100$ bandit problems sampled from $\mathcal{D}_{P}$ whereas the testing set contains another 10000 problems. When computing $\Delta\left(\pi_{\theta}\right)$, we estimate the regret for each of these problems by averaging results overs 100 runs. One calculation of $\Delta\left(\pi_{\theta}\right)$ thus involves simulating $10^{4}$ (resp. $10^{6}$ ) bandit episodes during training (resp. testing).

\subsection{Results}

Typical run of the learning algorithm. Figure 1 illustrates typical runs of Algorithm 2 when learning POWER-1. The curves represent the best regret achieved at each iteration of the EDA. The EDA optimizes the train regret and is not aware of the test regret score, which thus truly evaluates how well the learned policy generalizes to new bandit problems.
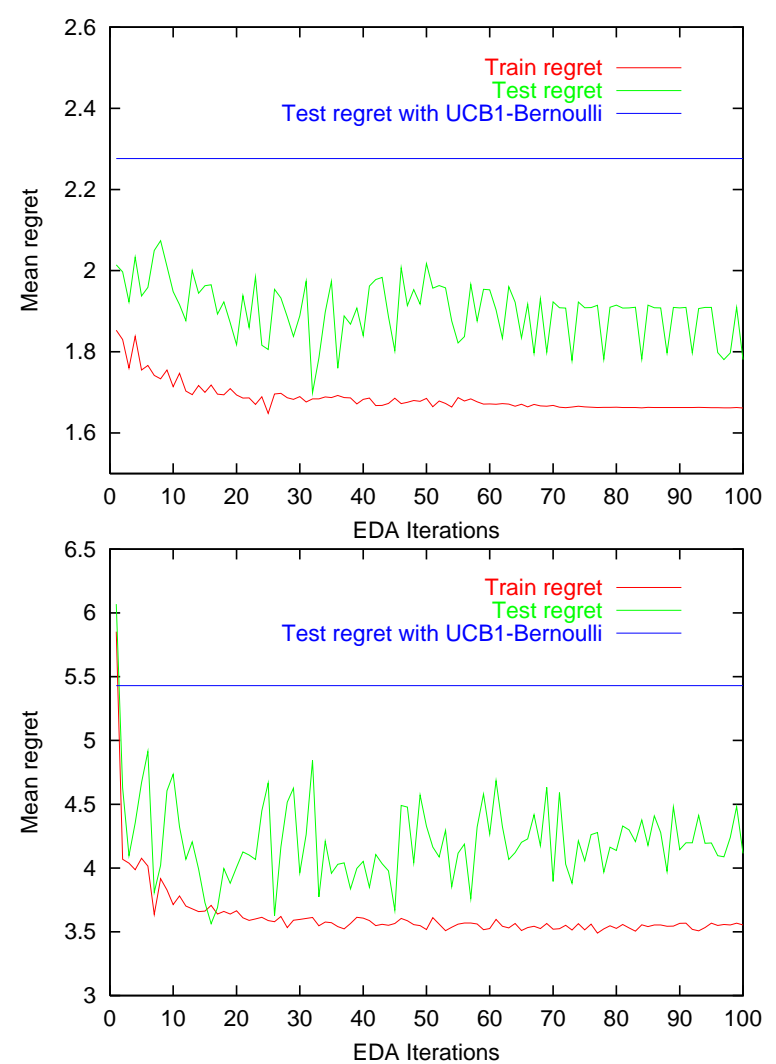

Figure 1: Training and testing regret of POWER-1 as a function of the number of EDA iterations. Left: training horizon 100. Right: training horizon 1000.

Note that to make things slightly faster, we only evaluated the test regrets roughly in this experiment, by doing a single run (instead of 100) per testing problem. See below for precise test regret values. As a matter of comparison, we also display the test regret achieved by the policy UCB1-BERNOULLI, which was especially designed for Bernoulli bandit problems.

We observe a common phenomenon of machine learning: the learned policy behaves slightly better on training problems than on testing problems. This effect could be reduced by using a larger set of training problems. However, it can be seen that the test regrets are already significantly better than those achieved by UCB 1-BERNOULLI. Furthermore, it can be seen that a few iterations are sufficient to find POWER-1 policies that outperform this baseline. For example, on the left curve, at iteration 1 the EDA test regret is already better then the baseline regret. This means that, in this experiment, sampling $8 d=128$ random POWER-1 policies was enough to find at least one policy outperforming UCB1-BERNOULLI.

We used a C++ based implementation to perform 


\begin{tabular}{|c|c|c|c|c|c|c|c|c|}
\hline \multirow[t]{2}{*}{ Policy } & Training & \multirow[t]{2}{*}{ Parameters } & \multicolumn{3}{|c|}{ Bernoulli } & \multicolumn{3}{|c|}{ Gaussian } \\
\hline & Horizon & & $\mathrm{T}=10$ & $\mathrm{~T}=100$ & $\mathrm{~T}=1000$ & $\mathrm{~T}=10$ & $\mathrm{~T}=100$ & $\mathrm{~T}=1000$ \\
\hline \multicolumn{9}{|c|}{ Untuned policies } \\
\hline UCB 1 & - & $C=2$ & 1.07 & 5.57 & 20.1 & 1.37 & 10.6 & 66.7 \\
\hline UCB 1-BERNOULLI & - & & 0.75 & 2.28 & 5.43 & 1.09 & 6.62 & 37.0 \\
\hline UCB 1-NORMAL & - & & 1.71 & 13.1 & 31.7 & 1.65 & 13.4 & 58.8 \\
\hline $\mathrm{UCB} 2$ & - & $\alpha=10^{-3}$ & 0.97 & 3.13 & 7.26 & 1.28 & 7.90 & 40.1 \\
\hline UCB-V & - & $c=1, \zeta=1$ & 1.45 & 8.59 & 25.5 & 1.55 & 12.3 & 63.4 \\
\hline KL-UCB & - & $c=0$ & 0.76 & 2.47 & 6.61 & 1.14 & 7.66 & 43.8 \\
\hline KL-UCB & - & $c=3$ & 0.82 & 3.29 & 9.81 & 1.21 & 8.90 & 53.0 \\
\hline$\varepsilon_{n}$-GREEDY & - & $c=1, d=1$ & 1.07 & 3.21 & 11.5 & 1.20 & 6.24 & 41.4 \\
\hline
\end{tabular}

\begin{tabular}{|c|c|c|c|c|c|c|c|c|}
\hline \multicolumn{9}{|c|}{ Tuned policies } \\
\hline \multirow{3}{*}{ UCB 1} & $\mathrm{~T}=10$ & $C=0.170$ & 0.74 & 2.05 & 4.85 & 1.05 & 6.05 & 32.1 \\
\hline & $\mathrm{T}=100$ & $C=0.173$ & 0.74 & 2.05 & 4.84 & 1.05 & 6.06 & 32.3 \\
\hline & $\mathrm{T}=1000$ & $C=0.187$ & 0.74 & 2.08 & 4.91 & 1.05 & 6.17 & 33.0 \\
\hline \multirow{3}{*}{ UCB 2} & $\mathrm{~T}=10$ & $\alpha=0.0316$ & 0.97 & 3.15 & 7.39 & 1.28 & 7.91 & 40.5 \\
\hline & $\mathrm{T}=100$ & $\alpha=0.000749$ & 0.97 & 3.12 & 7.26 & 1.33 & 8.14 & 40.4 \\
\hline & $\mathrm{T}=1000$ & $\alpha=0.00398$ & 0.97 & 3.13 & 7.25 & 1.28 & 7.89 & 40.0 \\
\hline \multirow{3}{*}{ UCB-V } & $\mathrm{T}=10$ & $c=1.542, \zeta=0.0631$ & 0.75 & 2.36 & 5.15 & 1.01 & 5.75 & 26.8 \\
\hline & $\mathrm{T}=100$ & $c=1.681, \dot{\zeta}=0.0347$ & 0.75 & 2.28 & 7.07 & 1.01 & 5.30 & 27.4 \\
\hline & $\mathrm{T}=1000$ & 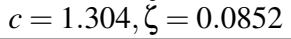 & 0.77 & 2.43 & 5.14 & 1.13 & 5.99 & 27.5 \\
\hline \multirow{3}{*}{ KL-UCB } & $\mathrm{T}=10$ & $c=-1.21$ & 0.73 & 2.14 & 5.28 & 1.12 & 7.00 & 38.9 \\
\hline & $\mathrm{T}=100$ & $c=-1.82$ & 0.73 & 2.10 & 5.12 & 1.09 & 6.48 & 36.1 \\
\hline & $\mathrm{T}=1000$ & $c=-1.84$ & 0.73 & 2.10 & 5.12 & 1.08 & 6.34 & 35.4 \\
\hline \multirow{3}{*}{$\varepsilon_{n}$-GREEDY } & $\mathrm{T}=10$ & $c=0.0499, d=1.505$ & 0.79 & 3.86 & 32.5 & 1.01 & 7.31 & 67.57 \\
\hline & $\mathrm{T}=100$ & $c=1.096, d=1.349$ & 0.95 & 3.19 & 14.8 & 1.12 & 6.38 & 46.6 \\
\hline & $\mathrm{T}=1000$ & $c=0.845, d=0.738$ & 1.23 & 3.48 & 9.93 & 1.32 & 6.28 & 37.7 \\
\hline
\end{tabular}

\begin{tabular}{|c|c|c|c|c|c|c|c|c|}
\hline \multicolumn{9}{|c|}{ Learned policies } \\
\hline \multirow{3}{*}{ POWER-1 } & $\mathrm{T}=10$ & & 0.72 & 2.29 & 14.0 & 0.97 & 5.94 & 49.7 \\
\hline & $\mathrm{T}=100$ & (16 parameters) & 0.77 & 1.84 & 5.64 & 1.04 & 5.13 & 27.7 \\
\hline & $\mathrm{T}=1000$ & $\ldots$ & 0.88 & 2.09 & 4.04 & 1.17 & 5.95 & 28.2 \\
\hline \multirow{3}{*}{ POWER-2 } & $\mathrm{T}=10$ & & 0.72 & 2.37 & 15.7 & 0.97 & 6.16 & 55.5 \\
\hline & $\mathrm{T}=100$ & (81 parameters) & 0.76 & 1.82 & 5.81 & 1.05 & 5.03 & 29.6 \\
\hline & $\mathrm{T}=1000$ & $\ldots$ & 0.83 & 2.07 & 3.95 & 1.12 & 5.61 & 27.3 \\
\hline
\end{tabular}

Table 1: Mean expected regret of untuned, tuned and learned policies on Bernoulli and Gaussian bandit problems. Best scores in each of these categories are shown in bold. Scores corresponding to policies that are tested on the same horizon $T$ than the horizon used for training/tuning are shown in italics.

our experiments. With 10 cores at $1.9 \mathrm{Ghz}$, performing the whole learning (and testing) of POWER-1 took one hour for $T=100$ and ten hours for $T=1000$.

Untuned, tuned and learned policies. Table 1 compares three classes of discrete bandit policies: untuned policies, tuned policies and learned policies. Untuned policies are either policies that are parameter-free or policies used with default parameters suggested in the literature. Tuned policies are the baselines that were tuned using Algorithm 2 and learned policies comprise POWER-1 and POWER-2. For each policy, we compute the mean expected regret on two kind of bandit problems: the 10000 testing problems drawn from $\mathcal{D}_{P}$ and another set of 10000 problems with a different kind of underlying reward distribution: truncated Gaussians to the interval $[0,1]$ (that means that if you draw a reward which is outside of this interval, you throw it away and draw a new one). In order to sample one such problem, we select the mean and the standard deviation of the Gaussian distributions uniformly in range $[0,1]$. For all policies except the untuned ones, we have used three different training horizons values $T=10, T=100$ and $T=1000$.

As already pointed out in (Auer et al., 2002), it can be seen that UCB1-BERNOULLI is particularly well fitted to bandit problems with Bernoulli distributions. It also proves effective on bandit problems with Gaussian distributions, making it nearly always outperform the other untuned policies. By tuning UCB 1, we outperform the UCB1-BERNOULLI policy (e.g. 4.91 instead of 5.43 on Bernoulli problems with $T=1000)$. This also sometimes happens with UCB-V. However, though we used a careful tuning 


\begin{tabular}{|c|cc|cc|cc|}
\hline Policy & \multicolumn{2}{|c|}{$\mathrm{T}=10$} & \multicolumn{2}{c|}{$\mathrm{T}=100$} & \multicolumn{2}{c|}{$\mathrm{T}=1000$} \\
& Regret & Win $\%$ & Regret & Win $\%$ & Regret & Win $\%$ \\
\hline UCB1-BERNOULLI & 0.75 & - & 2.28 & - & 5.43 & - \\
\hline UCB1 & 0.75 & $48.1 \%$ & 2.06 & $78.1 \%$ & 4.91 & $83.1 \%$ \\
UCB2 & 0.97 & $12.7 \%$ & 3.12 & $6.8 \%$ & 7.25 & $6.8 \%$ \\
UCB-V & 0.75 & $38.3 \%$ & 2.28 & $57.2 \%$ & 5.14 & $49.6 \%$ \\
KL-UCB & 0.73 & $50.5 \%$ & 2.10 & $65.0 \%$ & 5.12 & $67.0 \%$ \\
$\varepsilon_{n}$-GREEDY & 0.79 & $37.5 \%$ & 3.19 & $14.1 \%$ & 9.93 & $10.7 \%$ \\
\hline \multicolumn{7}{|c|}{ Learned policies } \\
\hline POWER-1 & $0.72 .6 \%$ & 1.84 & $82.3 \%$ & 4.04 & $91.3 \%$ \\
POWER-2 & 0.72 & $54.2 \%$ & 1.82 & $84.6 \%$ & 3.95 & $90.3 \%$ \\
\hline
\end{tabular}

Table 2: Regret and percentage of wins against UCB 1-BERNOULLI of tuned and learned policies.

procedure, UCB2 and $\varepsilon_{n}$-GREEDY do never outperform UCB1-BERNOULLI.

When using the same training and testing horizon $T$, POWER-1 and POWER-2 systematically outperform all the other policies (e.g. 1.82 against 2.05 when $\mathrm{T}=100$ and 3.95 against 4.91 when $T=1000)$.

Robustness w.r.t. the horizon $T$. As expected, the learned policies give their best performance when the training and the testing horizons are equal. When the testing horizon is larger than the training horizon, the quality of the policy may quickly degrade (e.g. when evaluating POWER- 1 trained with $T=10$ on an horizon $T=1000$ ), the inverse being less maked.

Robustness w.r.t. the kind of distribution. Although truncated Gaussian distributions are significantly different from Bernoulli distributions, the learned policies most of the time generalize well to this new setting and still outperform all the other policies.

Robustness w.r.t. to the metric. Table 2 gives for each policy, its regret and its percentage of wins against UCB1-BERNOULLI, when trained with the same horizon as the test horizon. To compute the percentage of wins against UCB1-BERNOULLI, we evaluate the expected regret on each of the 10000 testing problems and count the number of problems for which the tested policy outperforms UCB1BERNOULLI. We observe that by minimizing the expected regret, we have also reached large values of percentage of wins: $84.6 \%$ for $T=100$ and $91.3 \%$ for $T=1000$. Note that, in our approach, it is easy to change the objective function. So if the real applicative aim was to maximize the percentage of wins against UCB1-BERNOULLI, this criterion could have been used directly in the policy optimization stage to reach even better scores.

\section{CONCLUSIONS}

The approach proposed in this paper for exploiting a priori information for learning policies for $K$ armed bandit problems has been tested when knowing the time horizon and that arms rewards were generated by Bernoulli distributions. The learned policies were found to significantly outperform other policies previously published in the literature such as UCB1, UCB2, UCB-V, KL-UCB and $\varepsilon_{n}$-GREEDY. The robustness of the learned policy with respect to wrong information was also highlighted.

There are in our opinion several research directions that could be investigated for still improving the algorithm for learning policies proposed in this paper. For example, we found out that problems similar to the problem of overfitting met in supervised learning could occur when considering a too large set of candidate polices. This naturally calls for studying whether our learning approach could be combined with regularization techniques. More sophisticated optimizers could also be thought of for identifying in the set of candidate policies, the one which is predicted to behave at best.

The UCB1, UCB2, UCB-V, KL-UCB and $\varepsilon_{n^{-}}$ GREEDY policies used for comparison can be shown (under certain conditions) to have interesting bounds on their expected regret under asymptotic conditions (very large $T$ ) while we did not provide such bounds for our learned policy. It would certainly be relevant to investigate whether similar bounds could be derived for our learned policies or, alternatively, to see how the approach could be adapted so as to have learned policies that have strong theoretical performance guarantees. For example, better bounds on the expected regret could perhaps be obtained by identifying in a set of candidate policies the one that gives the smallest maximal value of the expected regret over this set rather than the one that gives the best average 
performances.

Finally, we suggest also to extend our policy learning scheme to other - and more complex exploration-exploitation problems than the one tackled in this paper, such as for example bandit problems where the arms are not statistically independent (Mersereau et al., 2009) or general Markov Decision processes (Ishii et al., 2002).

\section{REFERENCES}

Agrawal, R. (1995). Sample mean based index policies with $\mathrm{o}(\log \mathrm{n})$ regret for the multi-armed bandit problem. Advances in Applied Mathematics, 27:1054-1078.

Audibert, J., Munos, R., and Szepesvari, C. (2007). Tuning bandit algorithms in stochastic environments. Algorithmic Learning Theory (ALT), pages 150-165.

Audibert, J., Munos, R., and Szepesvari, C. (2008). Exploration-exploitation trade-off using variance estimates in multi-armed bandits. Theoretical Computer Science.

Auer, P., Fischer, P., and Cesa-Bianchi, N. (2002). Finitetime analysis of the multi-armed bandit problem. $M a$ chine Learning, 47:235-256.

Garivier, A. and Cappé, O. (2011). The KL-UCB algorithm for bounded stochastic bandits and beyond. CoRR, abs/1102.2490.

Gonzalez, C., Lozano, J., and Larrañaga, P. (2002). Estimation of Distribution Algorithms. A New Tool for Evolutionary Computation, pages 99-124. Kluwer Academic Publishers.

Ishii, S., Yoshida, W., and Yoshimoto, J. (2002). Control of exploitation-exploration meta-parameter in reinforcement learning. Neural Networks, 15:665-687.

Lai, T. and Robbins, H. (1985). Asymptotically efficient adaptive allocation rules. Advances in Applied Mathematics, 6:4-22.

Mersereau, A., Rusmevichientong, P., and Tsitsiklis, J. (2009). A structured multiarmed bandit problem and the greedy policy. IEEE Trans. Automatic Control, 54:2787-2802.

Pelikan, M. and Mühlenbein, H. (1998). Marginal distributions in evolutionary algorithms. In Proceedings of the International Conference on Genetic Algorithms Mendel '98, pages 90-95, Brno, Czech Republic.

Robbins, H. (1952). Some aspects of the sequential design of experiments. Bulletin of The American Mathematical Society, 58:527-536.

Rubenstein, R. and Kroese, D. (2004). The cross-entropy method : a unified approach to combinatorial optimization, Monte-Carlo simluation, and machine learning. Springer, New York.

Sutton, R. and Barto, A. (1998). Reinforcement Learning: An Introduction. MIT Press. 